\title{
Large-scale instability of a generalized turbulent Kolmogorov flow
}

\author{
B. Legras ${ }^{1}$ and B. Villone ${ }^{2}$ \\ ${ }^{1}$ Laboratoire de Méteorologie Dynamique, Ecole Normale Supérieure and CNRS (UMR8539), 24 rue Lhomond, 75231 Paris \\ Cedex 05, France \\ ${ }^{2}$ Istituto di Fisica dello Spazio Interplanetario di Torino, INAF,4 c. Fiume, 10133 Torino, Italy
}

Received: 22 May 2009 - Revised: 9 July 2009 - Accepted: 9 July 2009 - Published: 31 August 2009

\begin{abstract}
We present an analytical study of the large scale instability of a generalized turbulent Kolmogorov flow, i.e. a periodic shear flow where the molecular viscosity has been substituted by an eddy viscosity parameterized with the Clark-Smagorinsky model and where the external forcing is adapted to maintain the flow against this dissipation. We employ multiscaling technique assuming a scale separation between the basic scale of such a generalized turbulent Kolmogorov flow and the largest scales of the flow. The main result is that an amplitude equation for the large-scale secondary flow is obtained which exhibits, like for the standard Kolmogorov flow, an instability of the negative viscosity type. We find that the presence of mirror symmetry in the basic flow is a necessary condition and that further propagative and nonlinear contribution are produced otherwise. The result is encouraging for the generic existence of large-scale instabilities of the negative viscosity type in fully turbulent flows.
\end{abstract}

\section{Introduction}

Negative viscosity phenomenon has long been considered as a source of large-scale organized motion in geophysical and astrophysical flows (Starr, 1968). Large-scale instabilities of parallel shear flows are often considered as a paradigm for a number of astro-geophysical observed situations. The periodic Kolmogorov flow $U=\cos y$ is the simplest "toy" case belonging to this category. This flow exhibits a large-scale instability of the negative viscosity type for Reynolds number exceeding $R e=\sqrt{2}$ (Meshalkin and Sinai, 1961). For slightly supercritical conditions, the perturbation evolution is described by the Cahn-Hilliard equation (Nepomnyashchyi,

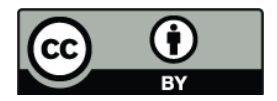

Correspondence to: $\mathrm{B}$. Legras

(legras@1md.ens.fr)
1976; Sivashinsky, 1985) whose solution is characterized by an inverse cascade of metastable states with scale growing in time (Kawasaki and Ohta, 1982; She, 1987). This cascade involves merging of jets until the gravest mode is reached. Intermediate states with multiple alternated jets may be stabilised by adding friction or by including the dispersive $\beta$ effect (Frisch et al., 1996; Manfroi and Young, 1999; Stuhne, 2001; Legras and Villone, 2003). Such stabilizing mechanisms have been advocated in the explanation of the features observed in the atmosphere of fast rotating Jovian planets, in the Antarctic circumpolar ocean current and other geophysical patterns (Rhines, 1994; Nozawa and Yoden, 1997; Huang and Robinson, 1998; Galperin et al., 2004; Hua et al., 2008). However, as a matter of fact, astro-geophysical fluids are characterized by very large Reynolds, whereas the critical Reynolds number relative to the large scale instability described by the Cahn-Hilliard equation is small. Manfroi and Young (2002) showed, studying the stability of the Kolmogorov flow on the $\beta$ plane, that in this case the critical Reynolds number may increase as a function of $\beta$ and of the angle between the direction of the Kolmogorov flow and the planetary vorticity gradient, but it stays still too low to be realistic. It is also known that friction can increase the critical Reynolds number (Obhukov, 1983; Dolzhansky, 1987; Thess, 1992; Burgess et al., 1999) but again this falls short of the conditions of geophysical flows at distance from boundary layers.

In this paper we address the problem of the large-scale instability of turbulent flows by investigating a generalized Kolmogorov flow instability, when the molecular viscosity is replaced by a parameterized eddy viscosity, for which we use the standard Clark-Smagorinsky model (Pope, 2000). Such a flow is maintained by a forcing term chosen in a way that the form of the parallel periodic shear flow is preserved in the high-Reynold parameterized regime. We denote such a flow as generalized turbulent Kolmogorov flow. Large eddy simulations (LES) of the fully turbulent regime of this flow

Published by Copernicus Publications on behalf of the European Geosciences Union and the American Geophysical Union. 
using the pure Smagorinsky model have been performed by (Woodruff et al., 1999, 2000). In our work, the large-scale instability is determined by multiscale analysis, from solvability conditions appearing in the perturbative expansion of the parameterized Navier-Stokes equation at successive orders. The novelty here is the nonlinear form of the dissipation as a representation of the effect of small-scale turbulence and as a model for high Reynolds flows.

This result is obtained at the price of a two scale separation hypothesis: the first one is between the small-scale turbulence represented by the Clark-Smagorinsky parameterization and the basic generalized Kolmogorov flow and the second one is between this basic flow and the large-scale of the searched instability. The method does not differ, in its principle, from that applied to the standard case of the Kolmogorov flow but is made much more complicated by the nonlinearities in the dissipation.

The paper is organised as follows. In Sect. 2, we describe the modification to the Navier-Stokes equation leading to the generalized Kolmogorov flow and we describe the perturbative expansion. In Sect. 3, we solve a case for which the calculations are amenable to a simple analytic form. In Sect. 4, we describe the solution of the full problem. In Sect. 5, we describe the numerical solution of the full problem and the results for three selected types of flow. Section 6 presents a discussion and the conclusions.

\section{The generalized turbulent Kolmogorov flow modelled with a Clark-Smagorinsky parameterization}

As in the standard problem (Sivashinsky, 1985), we use the framework of the two-dimensional incompressible NavierStokes equation. The filtered equation, in the sense of Large Eddy Simulation (LES)(Leonard, 1974), is

$\frac{\partial \bar{u}_{i}}{\partial t}+\frac{\partial}{\partial x_{j}}\left(\bar{u}_{i} \bar{u}_{j}+\tau_{i j}\right)=\frac{1}{\rho} \nabla \bar{p}+\mathcal{F}$,

where the overbar marks the filtered quantities, such as

$\bar{u}_{i}(\boldsymbol{x}, t)=\iint G(\boldsymbol{r}, \boldsymbol{x}) u_{i}(\boldsymbol{x}-\boldsymbol{r}, t) d^{2} \boldsymbol{r}$,

with $\iint G(\boldsymbol{r}, \boldsymbol{x}) d^{2} \boldsymbol{r}=1$.

The LES residual-stress tensor $\tau_{i j}$ is modelized as a turbulent dissipation according to the Clark-Smagorinsky model (Pope, 2000)

$\tau_{i j}=\frac{\Gamma^{2}}{12}\left(\frac{\partial \bar{u}_{i}}{\partial x_{k}} \frac{\partial \bar{u}_{j}}{\partial x_{k}}-\frac{1}{2} \frac{\partial \bar{u}_{l}}{\partial x_{k}} \frac{\partial \bar{u}_{l}}{\partial x_{k}} \delta_{i j}\right)-2 C_{s} \Gamma^{2} \bar{S} S_{i j}$,

where summation is performed over repeated indices,

$\bar{S}_{i j}=\frac{1}{2}\left(\frac{\partial \overline{u_{i}}}{\partial x_{j}}+\frac{\partial \overline{u_{j}}}{\partial x_{i}}\right)$ and $\bar{S}^{2}=2 \bar{S}_{i j} \bar{S}_{i j}$.
Here, $\Gamma$ is essentially the width of the filtering function $G$ and can be considered as the cutoff scale of the LES, and $C_{s}$ is an adjustable empirical constant. On the right hand side of Eq. (1), $\mathcal{F}$ is a forcing term, depending only on $y$, which maintains $u_{1}=U(y)$ as a stationary solution. This forcing acts at the scale of the flow $U(y)$ but contains also harmonics due to the nonlinear nature of the LES modelling. In the sequel we consider that all equations are non dimensionalized by two scales, $U_{0}$, the amplitude of $U(y)$ and $L$, its characteristic size in $y$. Then, $U(y)$ is assumed to be periodic with period $2 \pi$ and with zero mean over $[0,2 \pi]$. Although most of the calculations are done without any other assumption on $U(y)$, it will be shown that the large-scale instability is of negative viscosity type only if $U(y)$ has, up to an arbitrary translation in $y$, the mirror symmetry of an odd sine function in $y$ (Dubrulle and Frisch, 1991). This condition is easily obtained by assuming

$U(y)=\sin \theta(y)$,

$\theta(y)$ is chosen as

$\theta(y)=y+\sum_{p=1}^{\infty} \hat{\theta}_{p} \sin 2 p y$.

This profile generalizes the Kolmogorov flow for which $\theta(y)=y$ and allows for more complicated and realistic parallel wind profile than purely sinusoidal.

An important shortcoming of this parameterization within the scope of the present study is the vanishing of turbulent dissipation where velocity has an extrema. The undesirable consequence is the generation of spurious singularities in the singular perturbation problem to be studied here. In order to avoid this effect, we modify heuristically $\bar{S}$ as

$\bar{S}^{2}=2 \bar{S}_{i j} \bar{S}_{i j}+\mu\left({\overline{u_{1}}}^{2}+{\overline{u_{2}}}^{2}\right)$,

where $\mu$ is a positive constant. This modification accounts for the fact that the two-dimensional approximation is imperfect and can be seen as a contribution of the residual vertical shear to the strain rate, consistent with the ClarkSmagorinsky model. The dimensionalized form of $\mu$ would include a $1 / H^{2}$ factor where $H$ is a depth-scale of the flow. The precise form of the modification is not important as it contributes only by its value near the extrema of the generalized Kolmogorov flow and it is shown in Sect. 5 that the instability properties are weakly sensitive to the value of $\mu$.

Introducing the streamfunction $\psi$ such that $\overline{u_{1}}=\partial_{y} \psi, \overline{u_{2}}=-\partial_{x} \psi$, and taking the curl of Eq. (1), the basic equation is

$\partial_{t} \nabla^{2} \psi-\frac{\partial\left(\psi, \nabla^{2} \psi\right)}{\partial(x, y)}=K+\partial_{y} \mathcal{F}$ 
where $K$ is

$$
\begin{aligned}
& K=-\frac{\Gamma^{2}}{12}\left(\left(\partial_{x y^{3}} \psi+\partial_{x^{3} y} \psi\right)\left(\partial_{y y} \psi-\partial_{x x} \psi\right)\right. \\
& \left.+\partial_{x y} \psi\left(\partial_{x^{4}} \psi-\partial_{y^{4}} \psi\right)\right) \\
& +C_{s} \Gamma^{2}\left(4 \partial_{x y}\left(\bar{S} \partial_{x y} \psi\right)+\left(\partial_{y y}-\partial_{x x}\right) \bar{S}\left(\partial_{y y} \psi-\partial_{x x} \psi\right)\right)
\end{aligned}
$$

with

$$
\bar{S}^{2}=4\left(\partial_{x y} \psi\right)^{2}+\left(\partial_{x x} \psi-\partial_{y y} \psi\right)^{2}+\mu\left(\left(\partial_{x} \psi\right)^{2}+\left(\partial_{y} \psi\right)^{2}\right) .
$$

The streamfunction and all other primitives in $t$ defined in this work are assumed to have zero mean over $[0,2 \pi]$.

The main hypothesis of this work, following formally Meshalkin and Sinai (1961); Nepomnyashchyi (1976); Sivashinsky (1985), is the assumption of scale separation between the Kolmogorov flow and the large-scale secondary flow, and introduce slow variables $X=\epsilon x$ and $T=\epsilon^{2} t$ where $\epsilon$ is a small parameter. Then, it turns out that the flow depends only on $(X, y, T)$ and we can expand $\psi$ as

$$
\begin{aligned}
& \psi(X, y, t)=\Psi(y)+ \\
& \quad \psi_{0}(X, y, T)+\epsilon \psi_{1}(X, y, t)+\epsilon^{2} \psi_{2}(X, y, t)+\ldots,
\end{aligned}
$$

where $\Psi$ is the streamfunction for $U(y)$.

Equation (5) is then expanded in $\epsilon$ and the perturbation problem is solved at successive orders. It is easy to see that at leading order, the problem is reduced to

$\partial_{t} \partial_{y y} \psi_{0}=C_{s} \Gamma^{2} \partial_{y y}\left(\bar{S}_{0} \partial_{y y}\left(\Psi+\psi_{0}\right)\right)+\partial_{y} \mathcal{F}$,

with

$\bar{S}_{0}=\left[\left(\partial_{y y}\left(\Psi+\psi_{0}\right)\right)^{2}+\mu\left(\partial_{y}\left(\Psi+\psi_{0}\right)\right)^{2}\right]^{1 / 2}$,

which is always satisfied by

$\psi_{0}=\varphi_{0}(X, T)$

Hence, the leading perturbation depends on the slow and large-scale variables only. This property does not hold obviously for higher order perturbations. The remaining calculations at higher orders aim at obtaining an equation for the large-scale amplitude $\varphi_{0}(X, T)$, establishing a condition for the instability of the large-scale perturbation.

At each order $n$ in the expansion we need to solve

$\mathfrak{L}\left(\psi_{n}\right)=\mathcal{H}_{n}$,

where $\mathcal{L}$ is a linear operator and $\mathcal{H}_{n}$ holds for a complicated expression involving solutions to lower order equations in the perturbation expansion. As seen below, $\mathcal{L}$ satisfies $<\mathcal{L}(g)>=0$ for any function $g(y)$ with $<g>\equiv \frac{1}{2 \pi} \int_{0}^{2 \pi} g(y) d y$. Hence, a necessary solvability condition for Eq. (10) is given at each order by

$<\mathcal{H}_{n}>=0$.
The first order at which some terms appear that do not satisfy this condition provides the amplitude equation to be satisfied by $\varphi_{0}(X, T)$.

This problem has already been solved for the standard viscous dissipation in (Meshalkin and Sinai, 1961; Nepomnyashchyi, 1976; Sivashinsky, 1985) for a pure sinusoidal flow. Appendix A generalizes this derivation to a general parallel flow $U(y)$ without any assumption about symmetry. It is shown by Eqs. (A18,A19) that a large-scale instability of the negative viscosity type is obtained whatever is the profile of $U(y)$.

A first illustration of parameterized turbulence is provided in Appendix B where viscosity is replaced by hyperviscosity, a parameterization often used in pseudo-spectral simulations of turbulence or geophysical flows (Basdevant et al., 1981; Guermond and Prudhomme, 2003) which is still linear in the streamfunction but concentrates the dissipation into a small range of scales. The main result is that negative viscosity is still obtained but that there is no instability threshold due to the fact that hyperviscosity vanishes much more rapidly than viscosity in the large-scale limit. However, this parameterization hardly accounts for the nonlinear effects of small-scale turbulence.

\section{Instability for a simplified case}

When the parameterized dissipation Eq. (6), that involves a nonlinear dependence on the flow, replaces molecular viscosity, the complexity of algebra increases dramatically and the general calculation must be solved by symbolic calculations using Mathematica . The output of these calculations fills a large number of pages. Hence, we present here a simplified version where $\theta(y)=y$ in Eq. (3) and $\mu=1$ in Eq. (4) for which the flow is the standard sinusoidal Kolmogorov flow and $\bar{S}(\Psi)=1$.

However, the complete algebra, even for this simplified case, is still cumbersome and is better done using automatic symbolic calculation by Mathematica ${ }^{\mathrm{TM}}$. The corresponding notebook is provided as an electronic supplement to this paper (http://www.nonlin-processes-geophys.net/16/569/2009/ npg-16-569-2009-supplement.zip). We give here the results of the calculations at each order. The notation are simplified by noting $C=C_{s} \Gamma^{2}$ and $D=\Gamma^{2} / 12$ and using primes or parenthesized superscripts for the derivatives in $y$.

\subsection{First order}

At order 1, we obtain

$\mathfrak{L}\left(\psi_{1}\right)=\sin y \partial_{X} \varphi_{0}$,

with

$\mathfrak{L}(g)=\frac{C}{2}\left((3+\cos 2 y) g^{\prime \prime}+\sin 2 y g^{\prime}\right)^{\prime \prime}$, 
the solution of which is

$\psi_{1}=\frac{1}{C} \sin y \partial_{X} \varphi_{0}$.

In principle, a $\varphi_{1}(X, T)$ should be added to right hand side of Eq. (12). However, as we are only interested by the leading order amplitude equation for $\varphi_{0}(X, T)$, this is unnecessary and would only introduce additional spurious algebra (Bensoussan et al., 1978). It is always possible to assume that higher order corrections are contained within $\varphi_{0}(X, T)$ itself. Hence this term and similar terms in higher order equations are discarded.

\subsection{Second order}

At order 2, we obtain

$\mathfrak{L}\left(\psi_{2}\right)=\frac{3+C^{2}}{2 C} \cos y\left(\partial_{X} \varphi_{0}\right)^{2}-2 C \cos 2 y \partial_{X^{2}} \varphi_{0}$,

the solution of which is

$\psi_{2}=\frac{3+C^{2}}{4 C^{2}} \cos y\left(\partial_{X} \varphi_{0}\right)^{2}+f_{2}(y) \partial_{X^{2}} \varphi_{0}$,

with

$$
\begin{aligned}
f_{2}^{\prime}(y)= & \frac{1}{2} \sqrt{3+\cos 2 y}\left(F\left(y \mid \frac{1}{2}\right)-E\left(y \mid \frac{1}{2}\right) \frac{F\left(\frac{\pi}{2} \mid \frac{1}{2}\right)}{E\left(\frac{\pi}{2} \mid \frac{1}{2}\right)}\right) \\
& +\frac{1}{4} \frac{F\left(\frac{\pi}{2} \mid \frac{1}{2}\right)}{E\left(\frac{\pi}{2} \mid \frac{1}{2}\right)} \sin 2 y,
\end{aligned}
$$

where $F(y \mid m)$ and $E(y \mid m)$ are, respectively, the elliptic integrals of the first and second kind.

\subsection{Third order}

At order 3, we obtain

$$
\begin{aligned}
\mathfrak{L}\left(\psi_{3}\right)= & -\frac{1+C^{2}}{2 C^{2}} \sin y\left(\partial_{X} \varphi_{0}\right)^{3}-\frac{\sin y}{C} \partial_{X T} \varphi_{0} \\
& +a_{3 b} \partial_{X} \varphi_{0} \partial_{X^{2}} \varphi_{0}+a_{3 d} \partial_{X^{3}} \varphi_{0},
\end{aligned}
$$

with

$$
\begin{aligned}
a_{3 b} & =4 \cos 2 y f_{2}^{\prime}-(1-3 \cos 2 y) f_{2}^{(3)}+\sin 2 y\left(6+f_{2}^{(4)}\right) \\
& =-\left((1+\cos 2 y) f_{2}^{\prime}+\frac{1}{2} \sin 2 y\left(3-2 f_{2}^{\prime \prime}\right)\right)^{\prime \prime},
\end{aligned}
$$

and

$$
\begin{aligned}
a_{3 d}= & \frac{1}{4}\left(11 \sin y-9 \sin 3 y+4 \sin y\left(f_{2}+f_{2}^{\prime \prime}\right)\right) \\
& +D \cos y\left(f_{2}^{\prime}+f_{2}^{(3)}\right) \\
= & -\left(\frac{11}{4} \cos y-\frac{3}{4} \cos 3 y+\cos y f_{2}-\sin y f_{2}^{\prime}\right. \\
& \left.-D\left(\sin y f_{2}^{\prime}+\cos y f_{2}^{\prime \prime}\right)\right)^{\prime} .
\end{aligned}
$$

Hence the solvability condition is satisfied for all terms at order 3 and the solution is

$$
\begin{aligned}
\psi_{3}= & -\frac{1+C^{2}}{2 C^{3}} \sin y\left(\partial_{X} \varphi_{0}\right)^{3}+f_{3 b} \partial_{X} \varphi_{0} \partial_{X^{2}} \varphi_{0} \\
& +f_{3 d} \partial_{X^{3}} \varphi_{0}-\frac{1}{C^{2}} \sin y \partial_{X T} \varphi_{0},
\end{aligned}
$$

where $f_{3 b}(y)$ and $f_{3 d}$ are two functions which are solutions of $\mathfrak{L}\left(f_{3 b}\right)=a_{3 b}$ and $\mathfrak{L}\left(f_{3 d}\right)=a_{3 d}$.

\subsection{Fourth order}

At order 4, we obtain

$$
\begin{aligned}
\mathfrak{L}\left(\psi_{4}\right) & =-\frac{9+14 C^{2}+C^{4}}{16 C^{3}} \cos y\left(\partial_{X} \varphi_{0}\right)^{4} \\
& +a_{4 c}\left(\partial_{X} \varphi_{0}\right)^{2} \partial_{X^{2}} \varphi_{0}+a_{4 g} \partial_{X} \varphi_{0} \partial_{X^{3}} \varphi_{0} \\
& -\frac{7+C^{2}}{2 C^{2}} \cos y \partial_{X} \varphi_{0} \partial_{X T} \varphi_{0} \\
& +a_{4 l} \partial_{X^{4}} \varphi_{0}+\left(1+f_{2}^{\prime \prime}\right) \partial_{X^{2} T} \varphi_{0}+a_{4 o}\left(\partial_{X^{2}} \varphi_{0}\right)^{2} .
\end{aligned}
$$

The three terms $a_{4 c}, a_{4 g}$ and $a_{4 o}$ which depend on functions $f_{2}, f_{3 b}$ and $f_{3 d}$ are listed in Appendix C. They all satisfy the solvability condition and do not contribute to the amplitude equation.

Two terms, which are boxed in Eq. (14), do not satisfy the solvability condition. The coefficient in front of $\partial_{X^{2} T} \varphi_{0}$ provides a contribution -1 when averaged over the interval. The coefficient $a_{4 l}$ in front of $\partial_{X^{4}} \varphi_{0}$ is

$$
\begin{aligned}
a_{4 l}= & -C\left(\frac{1}{2} \cos 2 y+f_{2}^{\prime \prime}\right)-\left(\frac{3 C}{2}-\frac{D}{C} \cos ^{2} y-\frac{1}{C} \sin ^{2} y\right) \\
& -C\left(2 \cos 2 y f_{2}+\frac{3}{2} \sin 2 y f_{2}^{\prime}-\cos 2 y f_{2}^{\prime \prime}\right) .
\end{aligned}
$$

\subsection{Amplitude equation}

The second and the third term in the right hand side of Eq. (15) do not vanish after integration. Hence, after combining the contributions and integrating by part, the amplitude equation is obtained as

$\partial_{T} \varphi_{0}=\left(\frac{3 C}{2}-\frac{D}{2 C}-\frac{1}{2 C}+C I\right) \partial_{X^{2}} \varphi_{0}$,

with

$$
\begin{aligned}
I & =-\frac{3}{4 \pi} \int_{0}^{2 \pi} \sin 2 y f_{2}^{\prime} d y \\
& =-\frac{3}{4}\left(2-\frac{4}{3} \frac{F\left(\frac{\pi}{2} \mid \frac{1}{2}\right)}{E\left(\frac{\pi}{2} \mid \frac{1}{2}\right)}\right) \\
& =-0.12766 \ldots
\end{aligned}
$$


In terms of the parameters of the Clark-Smagorinsky model, the amplitude Eq. (16) exhibits a negative viscosity instability if

$$
\left(\frac{3}{2}+I\right) \Gamma^{2} C_{S}-\left(\frac{\Gamma^{2}}{12}+1\right) \frac{1}{2 \Gamma^{2} C_{s}}<0 .
$$

This inequality is satisfied if $\Gamma<\Gamma_{c}$ with

$$
\Gamma_{c}=\sqrt{\frac{\left(\frac{1}{12}+\sqrt{\frac{1}{144}+4 C_{s}^{2}(3+2 I)}\right)}{2(3+2 I) C_{s}^{2}}} .
$$

Using the standard value $C_{s}=0.008$ (Pope, 2000; Woodruff et al., 2000), we obtain $\Gamma_{c}=22.04$. For a pure Smagorinsky model $(D=0)$, the critical value would be $\Gamma_{c}=C_{S}^{-1 / 2}(3+2 I)^{-1 / 4}=8.69$. Using values of $C_{S}$ as large as 0.03 as suggested in Woodruff et al. (2000) when the grid size is getting close to the scale of the Kolmogorov flow yields $\Gamma_{c}=6.57$ for the Clark-Smagorinsky model and $\Gamma_{c}=4.49$ for the pure Smagorinsky model.

As soon as the filter width is smaller than $\Gamma_{c}$, a large-scale instability of the negative viscosity type is obtained. Since $\Gamma$ must be small with respect to the scale of the Kolmogorov flow, that is $2 \pi$ here, for consistency, this condition is always satisfied in practice.

\section{Full problem}

The calculations for the full problem are handled in the same way as for the simple problem but the calculations are then extremely heavy and cannot be presented here in detail. The notebook for the full case is provided as an electronic supplement (http://www.nonlin-processes-geophys.net/16/569/ 2009/npg-16-569-2009-supplement.zip) and we summarize here only the main results.

The linear operator is now

$\mathfrak{L}(g)=\left(\frac{\mu U^{2}+2 U^{\prime 2}}{\sqrt{U^{2}+U^{\prime 2}}} g^{\prime \prime}\right)^{\prime \prime}+\mu\left(\frac{U U^{\prime}}{\sqrt{U^{2}+U^{\prime 2}}} g^{\prime}\right)^{\prime \prime}$.

At order 1, we have

$\psi_{1}=\frac{1}{C} f_{1}(y) \partial_{X} \varphi_{0}$,

where $f_{1}$ is solution of

$\mathfrak{L}\left(f_{1}\right)=-U^{\prime \prime}$.

At order 2, we have

$\psi_{2}=f_{2 a}\left(\partial_{X} \varphi_{0}\right)^{2}+f_{2 b} \partial_{X^{2}} \varphi_{0}$, where we only need to know $f_{2 b}$ which is solution of

$$
\begin{aligned}
\mathfrak{L}\left(f_{2 b}\right)= & \left(\frac{\mu U^{2}+2 U^{\prime 2}}{\sqrt{\mu U^{2}+U^{\prime 2}}}\right)^{\prime \prime}+\frac{1}{C^{2}}\left(U f_{1}^{\prime}-U^{\prime} f_{1}\right)^{\prime} \\
& +\frac{D}{C^{2}}\left(U^{\prime} f_{1}^{\prime \prime}-U^{\prime \prime} f_{1}^{\prime}\right)^{\prime} .
\end{aligned}
$$

At order 3, seven terms are generated which all satisfy the solvability condition.

At order 4, the solvability condition provides again an amplitude equation displaying a parameterized viscosity

$\partial_{T} \varphi_{0}=v_{T} \partial_{X^{2}} \varphi_{0}$,

with

$$
\begin{aligned}
v_{T}= & -\frac{1}{C}<f_{1} U>+\frac{D}{C}<f_{1}^{\prime \prime} U> \\
& +C<\frac{\mu U^{2}+2 U^{\prime 2}}{\sqrt{\mu U^{2}+U^{\prime 2}}}>+C<f_{2 b}^{\prime} G(y)>,
\end{aligned}
$$

and

$G(y)=\frac{\left(U^{\prime 4}\right)^{\prime}+\mu U^{\prime 2}\left(3 U U^{\prime \prime}-U^{\prime 2}\right)}{2\left(\mu U^{2}+U^{\prime 2}\right)^{3 / 2}}$.

For most terms in the pertubative expansion, the solvability condition is obtained by finding a primitive in $y$ that does not depend on any assumption on $U(y)$. However, for a limited number of terms, it is necessary, unlike the pure viscous case discussed in Appendix A, to use the symmetry of $U(y)$ as defined in Eq. (3) in order to cancel the integral over $[0,2 \pi]$. It is the case, at third order, for the term in $\partial_{X^{3}} \varphi_{0}$. If the flow breaks the symmetry then our scaling is inappropriate and we need to assume $T=\epsilon t$. With this new scaling, a propagative equation is obtained at third order, and negative viscosity plus an additional nonlinear terms are obtained at fourth order. Namely, it can be shown (see accompanying notebook http://www.nonlin-processes-geophys.net/16/569/ 2009/npg-16-569-2009-supplement.zip) that the amplitude equation is then of the form:

$\partial_{T} \varphi_{0}+P \partial_{X} \varphi_{0}+\epsilon\left(Q\left(\partial_{X} \varphi_{0}\right)^{2}-v_{T} \partial_{X}^{2} \varphi_{0}\right)=0$,

where $P, Q$ are constants depending on the flow and $\nu_{T}$ is given by Eq. (22). In principle, the non linear term in Eq. (24) might be able to compensate for the instability induced by the negative viscosity. The study of these effects is not pursued in the sequel and is left for further work. However, we remark that the role of symmetries in obtaining negative viscosity effects is common feature in many instances (see, e.g. Dubrulle and Frisch, 1991 and Novikov and Papanicolau, 2001).

Notice that the condition $<U>=0$ is not a symmetry condition but it is merely consistent with the assumption of scale separation. Violation of this conditions leads only trivially to fast wave translation in the $X$ direction. 


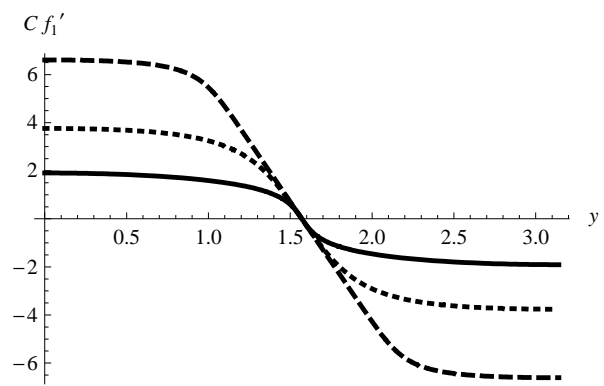

Fig. 1. $f_{1}^{\prime}(y)$ for Flow1 (solid), Flow2 (dash) and Flow3 (dotted) with $\mu=0.01$.

\section{Numerical solution of the full problem}

In order to estimate the contribution to the amplitude equation, it is necessary to solve the auxiliary problems Eqs. (20) and (21) for the two functions $f_{1}$ and $f_{2 b}$. These problems are actually simplified since the r.h.s. of Eq. (20) can be integrated twice and the terms on the r.h.s. of Eq. (21) can be integrated either once or twice, and we need only to know $f_{2 b}^{\prime}$. Hence, we only have to solve second order linear differential equations within the ensemble of periodic functions with zero mean over $[0,2 \pi]$. The problem for $f_{1}$ reduces to

$\mathfrak{L}_{1}\left(f_{1}^{\prime}\right)=-U \sqrt{U^{2}+U^{\prime 2}}$,

with

$\mathfrak{L}_{1}(g)=\left(\mu U^{2}+2 U^{\prime 2}\right) g^{\prime}+\mu U U^{\prime} g$.

With $U$ defined by Eq. (3), $f_{1}$ inherits the "odd sine" symmetry and Eq. (25) can be solved over the interval $\left[0, \frac{\pi}{2}\right]$ with the boundary conditions $f_{1}(0)=0$ and $f_{1}^{\prime}(\pi / 2)=0$. In turn, the problem for $f_{2 b}^{\prime}$ splits in three parts

$\mathfrak{L}_{1}\left(f_{2 b, 1}^{\prime}\right)=\mu U^{2}+2 U^{\prime 2}-k \sqrt{U^{2}+U^{\prime 2}}$,

$\mathfrak{L}_{2}\left(f_{2 b, 2}^{\prime}\right)=\left(U f_{1}^{\prime}-U^{\prime} f_{1}\right)$,

$\mathfrak{L}_{2}\left(f_{2 b, 3}^{\prime}\right)=\left(U^{\prime} f_{1}^{\prime \prime}-U^{\prime \prime} f_{1}^{\prime}\right)$,

with

$f_{2 b}^{\prime}=f_{2 b, 1}^{\prime}+\frac{1}{C^{2}} f_{2 b, 2}^{\prime}+\frac{D}{C^{2}} f_{2 b, 3}^{\prime}$,

and where

$\mathfrak{L}_{2}(g)=\left(\frac{\mu U^{2}+2 U^{\prime 2}}{\sqrt{U^{2}+U^{\prime 2}}} g^{\prime}+\mu \frac{U U^{\prime}}{\sqrt{U^{2}+U^{\prime 2}}} g\right)^{\prime}$.

The boundary conditions are $f_{2 b, i}^{\prime}(0)=f_{2 b, i}^{\prime}(\pi / 2)=0$, since $f_{2 b}$ exhibits "even cosine" symmetry, and $k$ is an integration constant. These problems are numerically solved with Mathematica $^{\mathrm{TM}}$ using NDSolve which provides the numerical solution of ordinary differential equations as a piewise cubic spline interpolating function. (a)

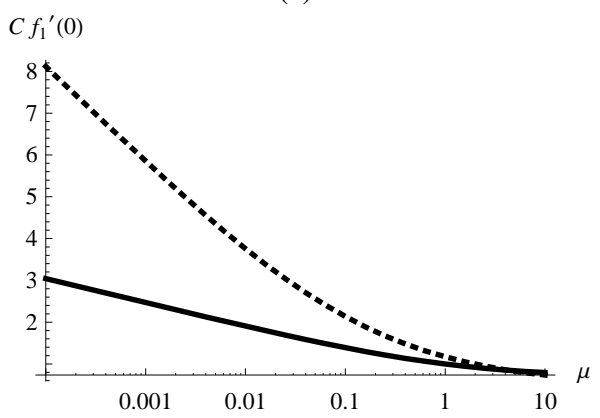

(b)

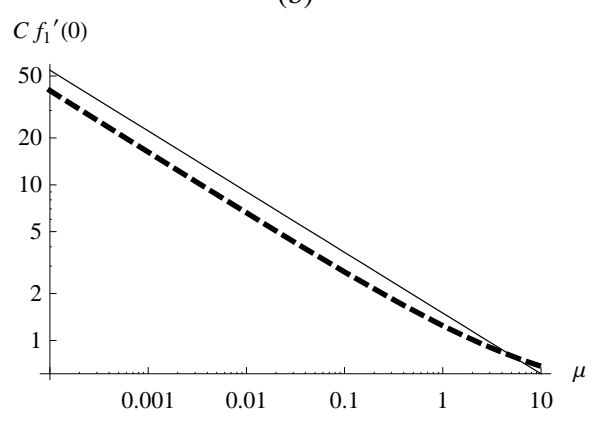

Fig. 2. (a) $f_{1}^{\prime}(y)(0)$ for Flow 1 (solid) and Flow 3 (dotted) as a function of $\mu$. (b) $f^{\prime}-1(y)(0)$ for Flow2 (dashed) and slope -0.39 (solid) as a function of $\mu$.

We consider three different velocity profile

- Flow1, with $U(y)=\sin y$, which is the standard sinusoidal profile of the Kolmogorov flow.

- Flow2, with $U(y)=\sin (y+1 / 2 \sin 2 y)$, which is a flow with a flat plateau such that the first five derivatives of $U(y)$ vanish in $\pi / 2$.

- Flow3, with $U(y)=\sin (y+1 / 4 \sin 4 y)$, which is an intermediate case between Flow1 and Flow2.

The details of the calculations can be checked in the accompanying notebook (http: //www.nonlin-processes-geophys.net/16/569/2009/ npg-16-569-2009-supplement.zip).

Figure 1 shows the functions $f_{1}^{\prime}$ for the three profiles and for $\mu=0.01$. It is apparent that the amplitude for Flow2 exceeds that of the two other flows and that $f_{1}^{\prime}$ exhibits, for this flow, a linear profile over a significant interval surrounding $\pi / 2$. The reason is that for all profiles $f_{1}^{\prime \prime}(\pi / 2)=-1 /(C \sqrt{\mu})$ but this value is preserved over an interval of order $O\left(1 / \mu^{1 / 5}\right)$ for Flow2 instead of $O(1 / \mu)$ for the other flows.

Figure 2 shows the value of $f_{1}^{\prime}(0)$ as a function of $\mu$ for the three profiles. The asymptotic behavior of Flow2 differs from the two others and is shown separately. It can be demonstrated that $f_{1}^{\prime}(0)$ scales as $-\log (\mu)$ for Flow1 and 
Flow 3 when $\mu$ is small, while it is visible that Flow 2 exhibits an anomalous algebraic scaling in $\mu^{-0.39}$.

At next order, the contribution $f_{2 b, 1}^{\prime}$ does not differ in amplitude between the three flows while the contributions $f_{2 b, 2}^{\prime}$ and $f_{2 b, 3}^{\prime}$ inherit the scaling properties of $f_{1}$.

After rearrangement of the terms, Eq. (22) is

$v_{T}=B_{1} C_{s} \Gamma^{2}+\frac{B_{2}}{C_{s} \Gamma^{2}}+\frac{B_{3}}{12 C_{s}}$,

with

$B_{1}=\left\langle\frac{\mu U^{2}+2 U^{\prime 2}}{\sqrt{U^{2}+U^{\prime 2}}}+f_{2 b, 1} G\right\rangle$,

$B_{2}=\left\langle-f_{1} U+f_{2 b, 2} G\right\rangle$,

$B_{3}=\left\langle-f_{1}^{\prime} U^{\prime}+f_{2 b, 3} G\right\rangle$.

It can be shown numerically that using the standard value $C_{S}=0.008, B_{1} B_{2}<0$ for all $\mu$ and hence, there is a threshold $\Gamma_{c}$ for negative viscosity defined as

$\Gamma_{c}=\frac{1}{C_{s}}\left(\frac{1}{2 B_{1}}\left(-B_{3}+\left(B_{3}^{2}-4 B_{1} B_{2} C_{s}^{2}\right)^{1 / 2}\right)^{1 / 2}\right.$.

Figure 3 shows the variation of $\Gamma_{c}$ as a function of $\mu$ for the three selected flows. It is visible that, although, $\Gamma_{c}$ tends to increase to larger values for small $\mu$, the range of values is the same for the three flows.

\section{Conclusions}

We have shown that a large-scale instability of the generalized turbulent Kolmogorov flow is obtained for parameterized LES equations as soon as the filtering length of the LES is smaller than a threshold that depends on the profile of the flow and of the added friction parameter $\mu$. The slow divergence when $\mu$ decreases suggests that negative viscosity is a robust feature that does not rely strongly on the fairly ad hoc addition of friction in Eq. (4).

The instability depends on the assumption that the basic flow has a mirror symmetry, a common feature to obtain negative viscosity (Dubrulle and Frisch, 1991).

Although, the parameterized LES equations are not equivalent to the Navier-Stokes equation at high Reynolds number (Woodruff et al., 1999), our results suggest that negative viscosity can be obtained in turbulent flow which exhibit an average zonal flow with the required symmetry and support the hypothesis that these negative-viscosity instabilities are important in geophysics. It would be interesting to look for such instabilities in numerical simulations.

Novikov and Papanicolau (2001); Novikov (2004) have shown, for Navier-Stokes equations, that cellular flows with a single scale can exhibit stabilization such that the large-scale instability is shifted to large Reynolds number. They mention that such effect is not observed with the Kolmogorov

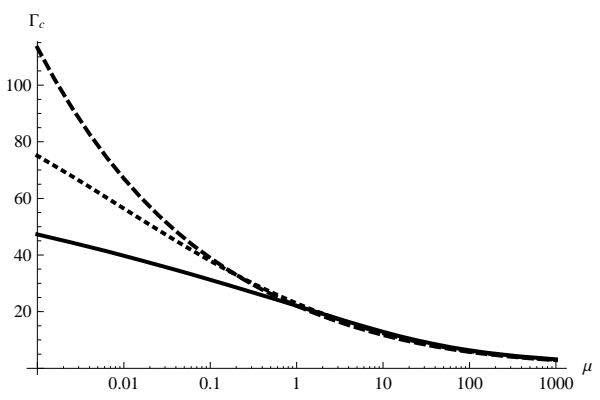

Fig. 3. $\Gamma_{c}$ for Flow1 (solid), Flow2 (dash) and Flow3 (dotted) with $C_{S}=0.008$.

flow and, indeed, we find that this is also true for the generalized turbulent Kolmogorov flow instability when the flow has mirror symmetry. However, nonlinear terms are generated in our calculation of the amplitude equation when this symmetry is broken. These terms are not obtained for pure viscous flow since the viscous Kolmogorov instability is generalized to any arbitrary parallel flow in Appendix A but may be seen as a consequence of the nonlinearity of the dissipation. It would be interesting to check whether such terms are able to stabilize and limit the scale of the instability.

\section{Appendix A}

\section{Transverse viscous instability of a general parallel flow}

We consider here the large-scale perturbation problem with dissipation performed by molecular viscosity $v$, i.e. when $K$ in Eq. (5) is replaced by $v \nabla^{4} \psi$, all the other terms being non modified. Derivatives in $y$ are noted using primes or parenthesized superscripts.

At order 0 , one obtains easily the condition $\nu \psi_{0}^{(4)}=0$ which is satisfied by choosing $\psi_{0}=\varphi_{0}(X, T)$.

At order 1, the perturbation $\psi_{1}$ is obtained by solving

$\mathfrak{L}\left(\psi_{1}\right)=-U^{\prime \prime} \partial_{X} \varphi_{0}$,

with

$\mathfrak{L}(g) \equiv \nu g^{(4)}$.

The solution is $\psi_{1}=f_{1} \partial_{X} \varphi_{0}$, with

$\mathfrak{L}\left(f_{1}\right)=-U^{\prime \prime}$.

At order 2, the solution is $\psi_{2}=f_{2 a}\left(\partial_{X} \varphi_{0}\right)^{2}+f_{2 b} \partial_{X^{2}} \varphi_{0}$, with

$\mathfrak{L}\left(f_{2 a}\right)=-f_{1}^{(3)}$,
$\mathfrak{L}\left(f_{2 b}\right)=F_{1}$, 
where $F_{i}=\left(U f_{i}^{\prime}-U^{\prime} f_{i}\right)^{\prime}$

At order 3 , the solution is

$\psi_{3}=f_{3 a}\left(\partial_{X} \varphi_{0}\right)^{3}+f_{3 b} \partial_{X^{2}} \varphi_{0}+f_{3 d} \partial_{X^{3}} \varphi_{0}+f_{3 g} \partial_{X T} \varphi_{0}$

with

$$
\begin{aligned}
& \mathfrak{L}\left(f_{3 a}\right)=-f_{2 a}^{(3)} \\
& \mathfrak{L}\left(f_{3 b}\right)=-f_{2 b}^{(3)}+2 F_{2 a}+G_{1,1} \\
& \mathfrak{L}\left(f_{3 d}\right)=U-2 v f_{1}^{\prime \prime}+F_{2 b} \\
& \mathfrak{L}\left(f_{3 g}\right)=f_{1}^{\prime \prime}
\end{aligned}
$$

where $G_{i, j}=\left(f_{i}^{\prime} f_{j}^{\prime}-f_{i} f_{j}^{\prime \prime}\right)^{\prime}$. All the terms on the r.h.s. of Eqs. (A6-A9) vanish by integration over $[0,2 \pi]$ and satisfy the solvability condition.

At order 4 , the solution is

$$
\begin{aligned}
\psi_{4}= & f_{4 a}\left(\partial_{X} \varphi_{0}\right)^{4}+f_{4 c} \partial_{X^{2}} \varphi_{0}\left(\partial_{X} \varphi_{0}\right)^{2} \\
& +\left(f_{4 g} \partial_{X^{3}} \varphi_{0}+f_{4 h} \partial_{X T} \varphi_{0}\right) \partial_{X} \varphi_{0} \\
& +f_{4 l} \partial_{X^{4}} \varphi_{0}+f_{4 n} \partial_{T X^{2}} \varphi_{0}+f_{4 o}\left(\partial_{X^{2}} \varphi_{0}\right)^{2}
\end{aligned}
$$

with

$$
\begin{aligned}
& \mathfrak{L}\left(f_{4 a}\right)=-f_{3 a}^{(3)} \\
& \mathfrak{L}\left(f_{4 c}\right)=-f_{3 b}^{(3)}+3 F_{3 a}+\left(3 f_{1}^{\prime} f_{2 a}^{\prime}-2 f_{2 a} f_{1}^{\prime \prime}-f_{1} f_{2 a}^{\prime \prime}\right)^{\prime} \\
& \mathfrak{L}\left(f_{4 g}\right)=-f_{3 d}^{(3)}-4 v f_{2 a}^{\prime \prime}+F_{3 b}+G_{2 b, 1} \\
& \mathfrak{L}\left(f_{4 h}\right)=-f_{3 g}^{(3)}+2 f_{2 a}^{\prime \prime} \\
& \mathfrak{L}\left(f_{4 l}\right)=-v+f_{1} U-2 v f_{2 b}^{\prime \prime}+F_{3 d} \\
& \mathfrak{L}\left(f_{4 m}\right)=f_{2 c}^{\prime \prime} \\
& \mathfrak{L}\left(f_{4 n}\right)=1+f_{2 b}^{\prime \prime}+F_{3 g} \\
& \mathfrak{L}\left(f_{4 o}\right)=-4 v f_{2 a}^{\prime \prime}+F_{3 b}+G_{1,2 b}
\end{aligned}
$$

The first two terms on the right hand side of Eq. (A14) and the first term on the r.h.s. of Eq. (A16) do not vanish by integration over $[0,2 \pi]$. They lead to the amplitude equation

$\partial_{T} \varphi_{0}-\left(\nu-<U f_{1}>\right) \partial_{X^{2}} \varphi_{0}=0$,

and hence to the critical viscosity for large-scale instability

$v_{c}=<\Psi^{2}(y)>^{1 / 2}$.

This expression generalizes that obtained by Meshalkin and Sinai (1961) with $U(y)=\sin y$. A Mathematica ${ }^{\mathrm{TM}}$ notebook for this calculation is accompanying this manuscript (http://www.nonlin-processes-geophys.net/16/569/2009/ npg-16-569-2009-supplement.zip).

\section{Appendix B}

\section{Instability in the presence of hyperviscosity}

Hyperviscosity is introduced by replacing $K$ in Eq. (5) by an iterated Laplacian of the streamfunction. We take here $K=v_{6} \nabla^{8} \psi$. Calculations for this case can be done in the same way as for the viscous dissipation in Appendix A. The remarkable fact is that the perturbative expansion is strikingly similar.

Up to order 2 , nothing is changed except that $\mathfrak{L}$ is now defined by $\mathfrak{L}(g) \equiv \nu_{6} g^{(8)}$.

At order 3, the only modification is to change the contribution $-2 v f_{1}^{\prime \prime}$ into $-4 v_{6} f_{1}^{(6)}$ in Eq. (A8).

At order 4, the Eqs. (A10, A11, A13, A15, A16) are unchanged. The contribution $-4 v f_{2 a}^{\prime \prime}$ is replaced by $-8 v_{6} f_{2 a}^{(6)}$. The most significant changes occur in Eq. (A14) where $-2 v f_{2 b}^{\prime \prime}$ is changed into $-4 v_{6} f_{2 b}^{(6)}$ but still does not contribute to the amplitude equation, and where $-v$ disappears. The reason is that the leading corresponding contribution in the hyperviscous expansion is in factor of $\partial_{X^{8}}$ and thus is $O\left(\epsilon^{8}\right)$

The consequence is that the amplitude equation is now

$\partial_{T} \varphi_{0}+<U f_{1}>\partial_{X^{2}} \varphi_{0}=0$

There is no more instability threshold and the negative viscosity is

$v_{T}=<U f_{1}>=\frac{<\xi^{2}(y)>}{v_{6}}$

where $\xi(y)$ is the third integral of $U(y)$ with zero-mean over $[0,2 \pi]$.

A Mathematica ${ }^{\text {TM }}$ notebook for this calculation is accompanying the manuscript (http: //www.nonlin-processes-geophys.net/16/569/2009/ npg-16-569-2009-supplement.zip)

\section{Appendix C}

\section{Fourth-order terms in the simplified case}

We list here, after rearrangement, the terms appearing in Eq. (14). Since they integrate all to zero over the interval $[0,2 \pi]$ the solvability condition is satisfied for these terms.

$$
\begin{aligned}
a_{4 c} & =\left(\frac{\left(5 C^{2}+19\right)}{8 C} \cos 2 y+\frac{C^{2}+3}{8 C}\left(3 \sin 2 y f_{2 b}^{\prime}\right.\right. \\
& \left.\left.+(3 \cos 2 y+1) f_{2 b}^{\prime \prime}\right)-2 \cos y\left(\cos y f_{3 b}^{\prime}-\sin y f_{3 b}^{\prime \prime}\right)\right)^{\prime \prime}
\end{aligned}
$$




$$
\begin{aligned}
a_{4 g} & =\frac{1}{C}\left(\sin y f_{2 b}+\cos y f_{2 b}^{\prime}\right)^{\prime}-\frac{D}{C}\left(\sin y f_{2 b}^{\prime \prime}-\cos y f_{2 b}^{\prime}\right)^{\prime} \\
& -\left(\frac{1}{8 C}(15 \cos y-7 \cos 3 y)-\frac{C}{8}(\cos 3 y-9 \cos y)\right. \\
& \left.+C \cos y f_{2 b}+(1+\cos 2 y) f_{3 d}^{\prime}-\sin 2 y f_{3 d}^{\prime \prime}\right)^{\prime \prime} \\
a_{4 o} & =3\left(\sin y\left(C \sin ^{2} y+\frac{1}{C}\left(\frac{D}{3}+1\right)\right)\right)^{\prime} \\
& -\frac{1}{C}\left(\sin y f_{2 b}^{\prime \prime}-\cos y f_{2 b}^{\prime}\right)^{\prime} \\
& -\frac{C}{4}\left(4 \sin ^{3} y f_{2 b}^{\prime}\left(f_{2 b}^{\prime \prime}-1\right)+2 \cos ^{3} y f_{2 b}^{\prime 2}\right. \\
& \left.-\frac{1}{2}(\cos 3 y-9 \cos y) f_{2 b}^{\prime \prime}\left(f_{2 b}^{\prime \prime}-2\right)\right)^{\prime \prime}
\end{aligned}
$$

Acknowledgements. This work has been supported by CNR/CNRS Project No. 11397.

Edited by: R. Grimshaw

Reviewed by: two anonymous referees

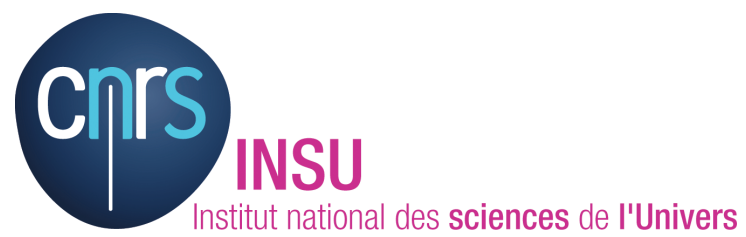

The publication of this article is financed by CNRS-INSU.

\section{References}

Basdevant, C., Legras, B., Sadourny, R., and Béland, M.: A study of barotropic model flows: intermittency, waves and predictability, J. Atmos. Sci., 38, 2305-2326, 1981.

Bensoussan, A., Lions, J.-L., and Papanicolau, G.: Asymptotic analysis of periodic structures, North Holland, Amsterdam, Netherlands, 1978.

Burgess, J. M., Bizon, C., McCormick, W. D., Swift, J. B., and Swinney, H. L.: Instability of the Kolmogorov flow in a soap film, Phys. Rev. E., 60, 715-721, 1999.

Dolzhansky, F. V.: On the influence of external friction on the stability of plane parallel flows of homogeneous incompressible fluids, Atmos. Ocean. Phys., 23, 262-268, 1987.

Dubrulle, B. and Frisch, U.: The eddy-viscosity of parity invariant flows, Phys. Rev. A, 43, 5355-5364, 1991.

Frisch, U., Legras, B., and Villone, B.: Large scale Kolmogorov flow on the beta-plane and resonant wave interaction, Physica D., 94, 36-56, 1996.

Galperin, B., Nakano, H., Huang, H. P., and Sukoriansky, S.: The ubiquitous zonal jets in the atmospheres of giants planets and Earth's oceans, Geophys. Res. Lett., 31, L13303, doi:10.1029/ 2004GL019691, 2004.

Guermond, J.-L. and Prudhomme, S.: Mathematical analysis of a spectral hyperviscosity LES model for the simulation of turbulent flows, ESAIM: Mathematical Modelling and Numerical Analysis, 37, 893-908, 2003.
Hua, B. L., dOrgeville, M., Fruman, M. D., Menesguen, C., Schopp, R., Klein, P., and Sasaki, H.: Destabilization of mixed Rossby gravity waves and the formation of equatorial zonal jets, J. Fluid Mech., 610, 311-341, 2008.

Huang, H.-P. and Robinson, W. A.: Two-dimensional turbulence and persistent zonal jets in a global barotropic model, J. Atmos. Sci., 55, 611-632, 1998.

Kawasaki, K. and Ohta, T.: Kink dynamics in one-dimensional nonlinear systems, Physica A, 116, 573, 1982.

Legras, B. and Villone, B.: Dispersion and friction-induced stabilization of the Cahn-Hilliard inverse cascade, Physica D, 175, 139-168, 2003.

Leonard, A.: Energy cascade in large eddy simulation of a turbulent fluid flow, Adv. Geophys., 18A, 237-248, 1974.

Manfroi, A. J. and Young, W. R.: Slow evolution of zonal jets on the beta plane, J. Atmos. Sci., 56, 784-800, 1999.

Manfroi, A. J. and Young, W. R.: Stability of $\beta$-plane Kolmogorov flow, Physica D, 162, 784-800, 2002.

Meshalkin, L. and Sinai, Y.: Investigation of the stability of a stationary solution of the system of equations for the plane movement of an incompressible viscous liquid, J. Appl. Math. Mech., 25, 1700-1705, 1961.

Nepomnyashchyi, A. A.: On the stability of the secondary flow of a viscous fluid in an infinite domain, Appl. Math. Mech., 40, 886891, 1976.

Novikov, A.: Eddy viscosity of cellular flows by upscaling, J. Comput. Phys., 195, 341-354, 2004.

Novikov, A. and Papanicolau, G.: Eddy viscosity of cellular flows, J. Fluid Mech., 446, 173-198, doi:10.1017/ S0022112001005730, 2001.

Nozawa, T. and Yoden, S.: Formation of zonal band structures in forced two-dimensional turbulence on a rotating sphere, Phys Fluids, 9, 2081-2093, 1997.

Obhukov, A. M.: Kolmogorov flow and laboratory simulation of it, Russ. Math. Surv., 38, 113-126, doi:10.1070/ RM1983v038n04ABEH004207, 1983.

Pope, S. B.: Turbulent Flows, Cambridge Univ. Press, 770 pp., 2000.

Rhines, P. B.: Jets, Chaos, 4, 313-339, 1994.

She, Z.: Metastability and vortex pairing in the Kolmogorov flow, Phys. Lett. A, 124, 161-164, 1987.

Sivashinsky, G.: Weak turbulence in periodic flows, Physica D., 17, 243-255, 1985.

Starr, V. P.: The physics of negative viscosity phenomenon, Earth and Planetary Sciences Series, Mc Graw Hill, New York, 1968.

Stuhne, G. R.: One-dimensional dynamics of zonal jets on rapidly rotating spherical shells, Physica D., 149, 43-79, 2001.

Thess, A.: Instability in two-dimensional spatially periodic flows. Part I: Kolmogorov flow, Phys. Fluids A., 4, 1385-1395, 1992.

Woodruff, S. L., Shebalin, J. V., and Hussaini, M. Y.: Directnumerical and Large-eddy Simulations of a Non-equilibrium Turbulent Kolmogorov Flow, Tech. Rep. 99-45, ICASE, NASA/CR-1999-209727, 1999.

Woodruff, S. L., Seiner, J. M., and Hussaini, M. Y.: Grid-size dependence in the Large-Eddy Simulation of Kolmogorov flow, AIAA Journal, 38, 600-604, 2000. 